\title{
Synthesis of NiO-CNTs nanocomposite for modification of glassy carbon electrode and Application for Electrochemical determination of fentanyl as an opioid analgesic drug
}

\author{
Hao Ding, Wei Tao* \\ Foundation Course Department, Wenhua College, Wuhan, 430074, China \\ "E-mail: tweiwhu@163.com
}

Received: 23 July 2021/ Accepted: 13 September 2021 / Published: 10 October 2021

\begin{abstract}
This study was focused on the synthesis of nanocomposite based on NiO and CNTs (NiO@CNTs) for modification of glassy carbon electrode (GCE) and application for electrochemical determination of fentanyl as an opioid analgesic drug. The NiO@CNTs nanocomposite was electrodeposited on the GCE surface. Results of structural analyses using SEM and XRD showed that the surface of the 3D of CNTs network was homogeneously covered by $\mathrm{NiO}$ nanoparticles, and the simultaneous electrodeposition of $\mathrm{NiO}$ nanoparticles and functionalized CNTs on GCE had been created a core-shell structure of NiO and CNTs with large void space between neighboring nanostructures. The results of electrochemical studies using CV and DPV techniques exhibited that the good conductivity of $\mathrm{NiO}$ and CNTs can contribute to the enhancement of the electrochemical response of the electrode toward fentanyl. The NiO@CNTs/GCE showed the selective and stable to determination fentanyl, and sensitivity, the limit of detection and linear range of $\mathrm{NiO} @ \mathrm{CNTs} / \mathrm{GCE}$ as fentanyl sensor were obtained $1.12219 \mu \mathrm{A} / \mu \mathrm{M}, 0.01$ $\mu \mathrm{M}$ and 10 to $160 \mu \mathrm{M}$, respectively. Moreover, a comparison between the obtained sensing results of $\mathrm{NiO} @ \mathrm{CNTs} / \mathrm{GCE}$ with reported fentanyl electrochemical sensor demonstrated comparable or better sensing properties and a wider linear range of NiO@CNTs/GCE than other reported electrochemical sensors. The results of the study indicated that the performance and precision of prepared electrodes for the analysis of fentanyl pharmaceutical and human serum samples showed acceptable precisions with appropriate recovery and RSD values. Therefore, of NiO@CNTs/GCE can be applied to accurate and reliable determination of fentanyl in clinical analyses.
\end{abstract}

Keywords: NiO@CNTs nanocomposite; Fentanyl; Clinical Samples; Differential pulse voltammetry; Selectivity

\section{$\underline{\text { FULL TEXT }}$}

(C) 2021 The Authors. Published by ESG (www.electrochemsci.org). This article is an open access article distributed under the terms and conditions of the Creative Commons Attribution license (http://creativecommons.org/licenses/by/4.0/). 